\title{
105. NOTAS SOBRE LA VEGETACIÓN DE ANDALUCÍA. III.
}

\author{
Baltasar CABEZUDO y Andrés V. PÉREZ LATORRE
}

Short notes about Andalusian vegetation. III.

Palabras clave. Vegetación, sintaxonomía, corología, Andalucía, España.

Key words. Vegetation, syntaxonomy, chorology, Andalusia, Spain.

1. Erico scopariae-Quercetum lusitanicae Rothmaler ex Br.- B1., P. Silva \& Rozeira 1965

Las comunidades caracterizadas por la quejigueta (Quercus lusitanica Lam.) en la Península Ibérica se restringen en territorio español a las provincias de Cádiz, Málaga y Huelva. Pérez Latorre et al. (1993:237, tab. 5) incluyen dichos quejigares enanos en la asociación Phyllireo-Quercetum fruticosae, creando una subasociación con Quercus rotundifolia para los inventarios onubenses, distinta de los gaditanos y malacitanos. A la vista de los últimos estudios geobotánicos en Portugal (Rivas Martínez et al., 1990) y el sudoeste de España (Pérez Latorre et al., 1996, 1999) parece más adecuado asignar las comunidades de $Q$. lusitanica de Huelva a la asociación portuguesa Erico scopariaeQuercetum lusitanicae. Las formaciones con quejigueta de Cádiz y Málaga difieren de las onubenses tanto en su composición florística como en la alianza en que se incluyen, ya que las primeras forman parte de brezales de Stauracanthenion boivinii y las segundas de formaciones arbustivas de Quercion fruticosae. La asociación Phyllireo-Quercetum fruticosae es por tanto exclusiva de territorios tingitanos (Marruecos) y la alianza Quercion fruticosae (en la Península Ibérica) de territorios portugueses y onubenses. En territorios algecireños (sector Aljíbico) Pérez Latorre et al. (1996:251) describen una comunidad arborescente de Quercus lusitanica e Ilex aquifolium con especies nemorales como Pedicularis lusitanica, Teucrium scorodonia subsp. baeticum, Senecio lopezii, Digitalis bocquetii y Brachypodium gaditanum que incluyeron provisionalmente en Quercetea ilicis y sin rango de asociación al no conocerse hasta el momento más que una localidad aislada.

\section{ESQUEMA SINTAXONÓMICO}

CALLUNO-ULICETEA Br.-B1. \& R. Tx. ex Klika \& Hadac 1944

+ Ulicetalia minoris Quantin 1935

* Ericion umbellatae Br.-Bl., P. Silva, Rozeira \& Fontes $1952 \mathrm{em}$. Rivas Martínez 1979

** Stauracanthenion boivinii Rivas Martínez 1979 (syn. Stauracanthion boivinii (Rivas Martínez 1979) Rivas Martínez, Fernández González \& Loidi, 1999)

Genisto tridentis-Stauracanthetum boivinii quercetosum lusitanicae Pérez Latorre, Nieto Caldera \& Cabezudo 1993 [Brezales aljíbicos con quejigo enano].

QUERCETEA ILICIS Br.-Bl. ex A. Bolòs 1950

+ Quercetalia ilicis Br.-Bl. ex Molinier 1934 em. Rivas Martínez 1975

* Quercion fruticosae Rothmaler 1954

Erico scopariae-Quercetum lusitanicae Rothmaler ex Br.- Bl., P. Silva \& Rozeira 1965 [Quejigares enanos luso-extremadurenses].

(syn. Phyllireo angustifoliae-Quercetum lusitanicae quercetosum rotundifoliae Pérez Latorre, Nieto Caldera \& Cabezudo 1993)

Phyllireo angustifoliae-Quercetum fruticosae Barbero, Quézel \& Rivas Martínez 1981 [Quejigares enanos tingitanos].

Comunidad de Quercus lusitanica e Ilex 
aquifolium in Pérez Latorre, Galán, Deil \& Cabezudo (1996) [Quejigares algecireños de quejigueta arborescente].

\section{Scrophulario laxiflorae-} Rhododendrion pontici Pérez Latorre, Galán de Mera \& Cabezudo nom. nov.

[Rhododendro pontici-Prunion lusitanicae Pérez Latorre, Galán de Mera \& Cabezudo 2000 non Rhododendro-Prunion lusitanicae Pérez Latorre, Galán de Mera \& Cabezudo 1999] Rhododendretalia pontici Pérez Latorre, Galán de Mera \& Cabezudo nom. nov.

[Rhododendro pontici-Prunetalia lusitanicae Pérez Latorre, Galán de Mera \& Cabezudo 2000 non Rhododendro-Prunetalia lusitanicae Pérez Latorre, Galán de Mera \& Cabezudo 1999]

De acuerdo con el artículo 31 del Código de Nomenclatura Fitosociológica vigente (Barkmann et al., 1986) se asigna un "nomen novum" para la alianza que reune la vegetación lauroide iberomarroquí-atlántica (Scrophulario laxiflorae-Rhododendrion pontici), puesto que el nombre anterior (Rhododendro-Prunion lusitanicae) ya había sido utilizado como sinónimo nomenclatural (D 10) de la alianza Osmundo-Alnion (Rivas-Martínez \& Sánchez Mata, 1999), al estar basado en la asociación Frangulo baeticae-Rhododendretum pontici Rivas Goday, Galiano \& Rivas-Martínez ex Rivas-Martínez, Fuente \& Sánchez Mata 1986 corr., que es ilegítima (art. 29, art. 37). El tipo nomenclatural de la alianza ScrophularioRhododendrion pontici continua por tanto siendo la asociación ScrophularioRhododendretum pontici (Pérez Latorre et al., 2000).

Del mismo modo, se asigna un nuevo nombre al orden que reúne la vegetación lauroide circummediterránea (Rhododendretalia pontici). El tipo nomenclatural de este orden pasa a ser la alianza ScrophularioRhododendrion pontici.

La creación de estos dos nombres nuevos además está basada en el uso anterior del homónimo heterotípico Rhododendro-Prunion lusitanicae (Pérez Latorre, Galán de Mera \& Cabezudo, 1999, 2000) según los artículos 31 y 39 .

Hasta el momento, es la clase PrunoLauretea azoricae la que en todos los sentidos (florístico, ecológico, ecomorfológico, paleofitogeográfico) mejor acoge estos bosquetes lauroides higrófilos de Rhododendron y no Quercetea ilicis como proponen Rivas Martínez \& Sánchez Mata (2000).

Respecto a la utilización del nombre Rhododendron ponticum L., consideramos que es el válido para la especie y por tanto para las poblaciones ibéricas, y no $R$. baeticum Boiss. $\&$ Reuter o su combinación subespecífica $R$. ponticum subsp. baeticum (Boiss. \& Reuter) Hand. Mazz., ambos meros sinónimos al no existir diferencias entre las poblaciones mediterráneo occidentales y orientales y ser el tipo $R$. ponticum L. de Algeciras (López González, 2001). La presencia de ojaranzales ibéricos y pónticos permite potenciar las similitudes de unas comunidades relictas (Rhododendretalia pontici) con un gran significado paleofitogeográfico en la región Mediterránea.

\section{ESQUEMA SINTAXONÓMICO}

PRUNO-LAURETEA AZORICAE Oberdorfer ex Rivas Martínez, Arnaiz, Barreno \& Crespo 1977 + Rhododendretalia pontici Pérez Latorre, Galán de Mera \& Cabezudo nom. nov.

* Scrophulario-Rhododendrion pontici Pérez Latorre, Galán de Mera \& Cabezudo nom. nov.

(Rhododendrenion baetici Rivas Martínez \& Sánchez Mata 2000)

Scrophulario laxiflorae-Rhododendretum pontici Pérez Latorre, Galán de Mera \& Cabezudo 2000 [Ojaranzales]

3. Arisaro proboscidei-Alnetum glutinosae Martínez Parras y Peinado 1987 (syn. Rhododendro pontici-Alnetum glutinosae 
(Rivas Goday \& Rivas Martínez in Rivas Martínez 1965) Rivas Martínez \& Sánchez Mata 2001)

Las alisedas aljíbicas han sufrido también la barahunda sintaxonómica de sus vecinos ojaranzales (Pérez Latorre et al., 2000). En una propuesta sorprendente para un territorio tan pequeño y tan homogéneo como los arroyos aljíbicos, Rivas Martínez y Sánchez Mata (2000) proponen la existencia de dos alisedas: la asociación ya hace tiempo descrita ArisaroAlnetum glutinosae (Martínez Parras y Peinado, 1987) y la que ahora proponen nomenclaturalmente como Rhododendro-Alnetum glutinosae.

La secuencia de los arroyos aljíbicos desde el nacimiento hasta su desembocadura en ríos mayores es la siguiente (Pérez Latorre et al.., 1996, 1999, 2000): 1. ojaranzal , 2. ojaranzal con alisos, 3. aliseda con ojaranzos, 4. aliseda con adelfas (ver esquema sintaxonómico).

El primero corresponde al ojaranzal de Scrophulario-Rhododendretum pontici. El segundo se trata de este mismo ojaranzal que se enriquece en alisos aislados o grupos de ellos cuando contacta con las alisedas, ocurriendo lo mismo cuando el biotopo ripario puntualmente (remansos, rezumaderos) favorece a Alnus glutinosa. El tercero es una aliseda con ojaranzos que ya está descrita como la subasociación típica alnetosum de ArisaroAlnetum glutinosae; la inexistencia de Rhododendron ponticum en los inventarios de Martínez Parras y Peinado puede ser debida a la escasez del área mínima inventariada (sólo $100 \mathrm{~m}^{2}$ ), puesto que en las dos localidades reseñadas (proximidad de Puerto de Galis y Arroyo del Tiradero) las alisedas siempre llevan ojaranzos (ver también Díez Garretas et al., 1988), que sólo desaparecen al menos parcialmente en el cuarto tramo, que corresponde a una aliseda con adelfas de Arisaro-Alnetum glutinosae nerietosum. Estos bosques riparios del Parque Natural de Los Alcornocales no se han salvado de la intervención humana, que consiste generalmente en favorecer la ganadería eliminando el estrato arbustivo y cargando las tintas sobre Rhododendron ponticum debido a su toxicidad para el ganado.

Como ejemplo de aliseda con ojaranzos de la proximidad del Puerto de Galis (Garganta del Medio, Jerez, Cádiz) damos a conocer el siguiente inventario: arroyo permanente, curso medio, $350 \mathrm{~m}$., cobertura $100 \%$, área $320 \mathrm{~m}^{2}(4$ x 80), orientación norte. Características: Alnus glutinosa 4, Arisarum proboscideum 3, Athyrium filix-femina + , Osmunda regalis + , Sibthorpia europaea + , Rhododendron ponticum 2, Frangula alnus subsp. baetica 2, Laurus nobilis +. Compañeras: Hedera helix subsp. canariensis +, Davallia canariensis +, Ficus carica + , Quercus canariensis 1, Rubia agostinhoi + , Asplenium onopteris 1, Smilax aspera var. altissima + , Rubia peregrina + , Ruscus aculeatus +, Clematis cirrhosa +, Viburnum tinus 1, Phillyrea latifolia 1, Phillyrea angustifolia + , Digitalis bocquetii + , Rubus ulmifolius + , Lonicera periclymenum subsp. hispanica 2, Carex acuta + , Oenanthe crocata + , Ranunculus ficaria 1, Allium triquetrum + , Tamus communis + .

\section{ESQUEMA SINTAXONÓMICO}

(Los números corresponden a los distintos tramos riparios comentados en el texto)

PRUNO-LAURETEA AZORICAE Oberdorfer ex Rivas Martínez, Arnaiz, Barreno \& Crespo 1977 + Rhododendretalia pontici Pérez Latorre, Galán de Mera \& Cabezudo nom. nov.

* Scrophulario-Rhododendrion pontici Pérez Latorre, Galán de Mera \& Cabezudo nom. nov. (syn. Rhododendrenion baetici Rivas Martínez \& Sánchez Mata 2000)

Scrophulario laxiflorae-Rhododendretum pontici Pérez Latorre, Galán de Mera \& Cabezudo 2000 [Ojaranzales] (1)

Var. de Alnus glutinosa [Variante hidrófila con alisos] (2) 
QUERCO-FAGETEA Br.-Bl. \& Vlieger in Vlieger 1937

+ Populetalia albae Br.-Bl. ex Tchou 1948

* Osmundo-Alnion (Br.-Bl., P. Silva \& Rozeira 1956) Dierschke \& Rivas Martínez in Rivas Martínez 1975

Arisaro proboscidei-Alnetum glutinosae Martínez Parras \& Peinado Lorca 1987

alnetosum glutinosae (syn. Rhododendro ponticiAlnetum glutinosae (Rivas Goday \& Rivas Martínez in Rivas Martínez 1965) Rivas Martínez \& Sánchez Mata 2001) [Alisedas aljíbicas] (3)

nerietosum oleandri Martínez Parras \& Peinado Lorca 1987 [Variante con adelfas] (4)

\section{BIBLIOGRAFÍA}

BARKMAN, J. J., J. MORAVEC \& S. RAUSCHERT -1986- Código de nomenclatura fitosociológica (Trad. J. IZCO \& M.J. ARCO-AGUILAR, 1988). Opusc. Bot. Pharm. Complutensis 4: 9-74.

DÍEZ GARRETAS, B., J. CUENCA y A. ASENSI 1986- Datos sobre la vegetación del subsector Aljíbico (provincia Gaditano-OnuboAlgarviense). Lazaroa 9: 315-332.

LOPEZ GONZÁLEZ, G. -2001- Los árboles y arbustos de la Península Ibérica e Islas Baleares. Ed. Mundi Prensa. Madrid.

MARTINEZ PARRAS, J.M. y M. PEINADO LORCA -1987-Datos sobre la vegetación riparia del sector Gaditano. Secret. Public. ser. Informes 22: 199-206. Universidad de La Laguna.

PÉREZ LATORRE, A. V., J. M. NIETO CALDERA y B. CABEZUDO -1993-Contribución al conocimiento de la vegetación de Andalucía. II. Los alcornocales. Acta Bot. Malacitana 18: 223258.

PÉREZ LATORRE, A.V., A. GALÁN DE MERA, U. DEIL y B. CABEZUDO - 1996- Fitogeografía y vegetación del sector Aljíbico (Cádiz-Málaga, España). Acta Bot. Malacitana 21: 241-267.

PÉREZ LATORRE, A. V., A. GALÁN DE MERA, P. NAVAS, D. NAVAS, Y. GIL y B. CABEZUDO -1999- Datos sobre la flora y vegetación del Parque Natural de los Alcornocales (Cádiz-Málaga, España). Acta Bot. Malacitana 24:133-184.
PÉREZ LATORRE, A. V., A. GALÁN DE MERA y B. CABEZUDO -2000- La vegetación caracterizada por Rhododendron ponticum $\mathrm{L}$. En Andalucía (España). Una complicada historia nomenclatural para una realidad fitocenológica. Acta Bot. Malacitana 25:198-205

RIVAS-MARTINEZ, S., M. LOUSA, T.E. DIAZ, F.FERNANDEZ GONZALEZ \& J.C. COSTA 1990- La vegetación del sur de Portugal. Itinera Geobot. 3: 5-126.

RIVAS MARTÍNEZ y SÁNCHEZ MATA -1999Rhododendro pontici-Prunion lusianicae (Pruno-Lauretea azoricae) nomenclaturalmente sinónimo de Osmundo-Alnion (QuercoFagetea). Stud. Bot. 18:157-159.

RIVAS MARTÍNEZ, S. y D. SÁNCHEZ MATA 2000- Precisiones sintaxonómicas sobre las alisedas aljíbicas. Lazaroa 21:150-152.

Aceptado para su publicación en septiembre de 2001

Dirección de los autores. Departamento de Biología Vegetal. Facultad de Ciencias. Univesidad de Málaga. Campus de Teatinos. 29071-Málaga. 